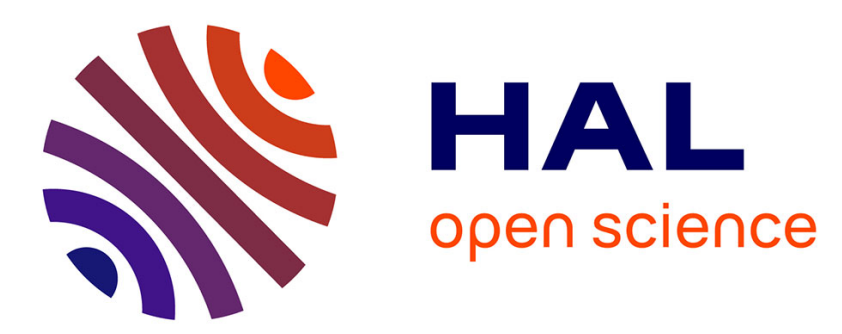

\title{
PID Control Design for First-Order Delay Systems via MID Pole Placement: Performance vs. Robustness
}

\author{
Dan Ma, Islam Boussaada, Jianqi Chen, Catherine Bonnet, Silviu-Iulian \\ Niculescu, Jie Chen
}

\section{- To cite this version:}

Dan Ma, Islam Boussaada, Jianqi Chen, Catherine Bonnet, Silviu-Iulian Niculescu, et al.. PID Control Design for First-Order Delay Systems via MID Pole Placement: Performance vs. Robustness. Automatica, 2021, 10.1016/j.automatica.2021.110102 . hal-03427550

\author{
HAL Id: hal-03427550 \\ https://hal.science/hal-03427550
}

Submitted on 14 Nov 2021

HAL is a multi-disciplinary open access archive for the deposit and dissemination of scientific research documents, whether they are published or not. The documents may come from teaching and research institutions in France or abroad, or from public or private research centers.
L'archive ouverte pluridisciplinaire $\mathbf{H A L}$, est destinée au dépôt et à la diffusion de documents scientifiques de niveau recherche, publiés ou non, émanant des établissements d'enseignement et de recherche français ou étrangers, des laboratoires publics ou privés. 


\title{
PID Control Design for First-Order Delay Systems via MID Pole Placement: Performance vs. Robustness
}

\author{
Dan $\mathrm{Ma}^{\mathrm{a}}$ Islam Boussaada ${ }^{\mathrm{b}, \mathrm{d}}$ Jianqi Chen ${ }^{\mathrm{c}}$ Catherine Bonnet ${ }^{\mathrm{b}}$ \\ Silviu-Iulian Niculescu ${ }^{\mathrm{b}}$ Jie Chen ${ }^{\mathrm{c}}$

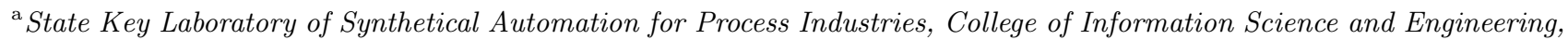 \\ Northeastern University, Shenyang, China, \\ madan@mail.neu.cn.edu \\ ${ }^{\mathrm{b}}$ Université Paris Saclay, CNRS-CentraleSupelec, Inria Saclay, Laboratoire des Signaux et Systèmes (L2S), 3 rue Joliot-Curie \\ 91192 Gif-sur-Yvette cedex (France) (e-mail: \\ Islam.Boussaada@12s.centralesupelec.fr, catherine.bonnet@centralesupelec.fr, \\ Silviu.Niculescu@l2s. centralesupelec.fr \\ ${ }^{\mathrm{c}}$ Department of Electrical Engineering, City University of Hong Kong, Hong Kong, China, \\ jianqchen2-c@my.cityu.edu.hk, jichen@cityu.edu.hk \\ ${ }^{\mathrm{d}}$ Institut Polytechnique des Sciences Avancées (IPSA), Bis, 63 Boulevard de Brandebourg, 94200 Ivry-sur-Seine (France)
}

\section{(To appear in: Automatica)}

\begin{abstract}
The PID control is favored in controlling industrial processes for its ease of implementation. In this paper we present an analytical study, with an aim at stability robustness, tracking performance, and pole placement of first-order unstable plants under PID control. We employ a multiplicity-induced dominancy (MID) strategy to design and tune PID controller gains, which is shown to possess desirable properties that ensure specified decay rate of the closed-loop response and the stability robustness of the closed-loop system in spite of variations in the delay parameter. We also study the tradeoff between delay robustness and tracking performance of PID controllers. We show that under the constraint of steady-state tracking, the maximal delay robustness range can be computed by solving a unimodal pseudo-concave optimization problem.
\end{abstract}

Key words: Time-delay systems, PID control, delay robustness, pole placement, steady-state tracking.

\section{INTRODUCTION}

A canonical case of study for PID (proportional-integralderivative) control is the first-order delay system

$$
P_{\tau}(s)=\frac{K}{1+T s} e^{-\tau s},
$$

where $K>0$ is the plant gain, $\tau \geq 0$ represents the time delay in the controlled process, and $T \geq 0$ is referred to as the time constant of the system. Traditionally, the implementation of a PID controller is done via parameter tunning, based on such empirical rules as Ziegler-Nichols method and its offsprings (Åström and Hägglund, 1995). The PID control has been long well-known for its simplicity, ease of implementation and cost-effectiveness. Even in a post-modern and information-centric era of present time, it remains to stand out as a most favored control technology and dominates industrial control systems; by documented statistics, more than $95 \%$ of the physical-layer control loops are still enabled by PID controllers, with proven, widespread acceptance by the industrial control community (Åström and Hägglund, 1995; Samad, 2017).

For its recognized advantages, there has been sustained interest in PID control. Some of the recent work on PID control has been focused on analytical studies, which have led to new findings, improved design and tuning rules on e.g., time delay systems, nonlinear systems, and 
extensions to multi-agent systems (Xu et al., 2013; Ma et al., 2020b). Specifically, steady-state tracking performance was examined in Åström and Hägglund (1995); Silva et al. (2002), the delay robustness problems were studied in Silva et al. (2002); Ma and Chen (2019); Chen et al. (2019), and nonlinear regulation problems were considered in Zhao and Guo (2017). Of particular relevance to this paper, there is a long stream of works concerning design problems such as pole placement of timedelay systems by PID controllers, based on tools ranging from algebraic techniques (Wang et al., 2017; Emre and Mehmet, 2018; Das et al., 2020), numerical computation (Michiels et al., 2002; Pavel et al., 2013; Firouzbahrami and Nobakhti, 2017; Jaromír and Pavel, 2019; Zhang et al., 2020), to performance and robustness considerations (Srivastava and Pandit, 2016; Tavakoli and Safaei, 2018; Rao et al., 2020).

Also of close pertinence are studies on open-loop unstable systems with time delays. Physical processes that can be suitably modeled as an unstable delay plant are widely found in the process industry, including, e.g., bioreactors and tank fermenters (see, e.g, O'Dwyer (2013) and the references therein). For such systems, the delay margin (Middleton and Miller, 2007) furnishes an intrinsic measure of robustness with respect to variations in time delay, defined as the maximal range of time delay within which the system can be stabilized robustly by a fixed controller. This notion has been extensively studied recently, based on different methods and techniques (see, e.g., Bresch-Pietri et al. (2012); Gaudette and Miller (2016); Foias et al. (1996); Ju and Zhang (2016); Miller and Davison (2005); Zhong (2006); Zhou et al. (2009, 2012)). Analytical and computational results have been previously obtained for determining the delay margin achievable by PID controllers (Silva et al., 2002; Ma and Chen, 2019; Chen et al., 2019). This fundamental limit, however, is attained with no regard to other control objectives, and hence is in inherent conflict with a system's transient and steady-state performance. In a more realistic design, one must then incorporate performance objectives and address the tradeoff between performance and robustness. This motivates our work in this paper.

We study the analytical design of PID controllers for delay robustness and performance. We consider first-order unstable plants. This consideration partly stems from the fact that industrial processes are often modeled by first- and generally low-order systems, partly due to the limitation of PID controllers in controlling high-order dynamics. While limited in scope, the study of first-order plants does allow an in-depth analysis, availing analytical results that render useful insights. We develop a poleplacement design via the so-called multiplicity-induced dominancy (MID), which seeks to place the dominant poles of the closed-loop system at a same location, resulting in a multiple dominant pole. The design consequently ensures such transient property as the degree of stability, or alternatively the decay rate of the closed- loop response. Of equal importance, it also renders a certain amount of delay robustness, with a guaranteed range of delay within which the plant can be robustly stabilized. Interestingly, the result also demonstrates a clear tradeoff between the delay robustness range and the decay rate, and in the limit the range approaches to the delay margin, i.e., the largest range possible to maintain robust stability, whilst the closed-loop system becomes marginally stable.

The analytical MID pole placement design for time-delay systems by itself poses a nontrivial problem, even for a first-order plant. The technical intricacy is further compounded by additional requirement to ensure delay robustness. Previously an analytical proof of the dominancy of a spectral value for a scalar state-space equation with a single delay was presented in Hayes (1950). The dominancy property was also established for scalar state-space delay equations in Boussaada et al. (2016), and further exploited in Boussaada et al. (2018, 2020) for second-order retarded differential equations. For more general pole placement problems via PID control, numerical optimization-based methods developed in (Vanbiervliet et al., 2008; Ramirez et al., 2015) incorporated as well the multiplicity-induced dominancy. It is useful to note that in all the aforementioned results, the assumption of a retarded system is critical. In an important distinction, however, with the use of PID control, the system under consideration in the present paper constitutes invariably a neutral delay system. Unlike retarded systems which admit a finite number of roots in any right half plane, neutral delay systems may have infinitely many roots located in the open right half plane. This makes our design considerably more challenging and it requires a nontrivial extension of the multiplicityinduced dominancy analysis to neutral delay systems.

We also investigate the tradeoff between the delay margin and the steady-state tracking performance. While in the limit the delay margin can be analytically determined for first-order systems, under performance constraint it generally poses a constrained parametric optimization problem which in turn requires a brute-force search in the three design parameters of the PID controller. Nevertheless, we show that under a prescribed specification of tracking error, the problem can be solved computationally as a unimodal pseudo-concave program, i.e., a univariate smooth convex optimization problem. As such, under the steady-state tracking objective, the maximal delay robustness range can be computed using convex programming methods, or gradient-based bisection methods. The attainable range is found to be inversely proportional to the tracking accuracy. The solution is made possible by recasting the problem as one of nonlinear programming, and tackled by employing the well-known Fritz John condition.

The remaining paper is organized as follows. In Section 2 we introduce the mathematical background required 
in the sequel, together with the notion and facts of delay margin. We then provide the multiplicity-induced dominancy design in Section 3, where we analyze the rightmost root of the closed-loop quasipolynomial in details. The multiplicity and dominance of the rightmost root is established. In Section 4, we further establish the delay robustness of this multiplicity-induced dominancy design, showing that a range of delay exists that guarantees the closed-loop stability. Section 5 studies the tradeoff between tracking performance and delay robustness. We show that under the constraint of steady-state tracking, the maximal delay robustness range can be computed by solving a unimodal pseudo-concave optimization problem. An illustrative example is then provided in Section 6 , and the paper concludes in Section 7.

Partial results of this paper were previously presented in Chen and Chen (2020); Ma et al. (2020a); the current paper includes updated results with full proofs.

\section{Preliminary background}

\subsection{Mathematical preliminaries}

The primary technical machineries to be used in the sequel dwell on complex variables theory and mathematical programming problems. Consider the complex function

$$
G(s)=\frac{r(s)}{Q_{0}(s)+Q_{\tau}(s) \mathrm{e}^{-s \tau}},
$$

where $r(s), Q_{0}(s)$, and $Q_{\tau}(s)$ are polynomials with degrees satisfying the condition $\operatorname{deg}(r) \leq \operatorname{deg}\left(Q_{0}\right)=$ $\operatorname{deg}\left(Q_{\tau}\right)$, and $\tau \geq 0$ is a constant. We first state the following result from Partington and Bonnet (2004), which gives an explicit localization of the spectrum chain's asymptote for quasipolynomials of neutral systems.

Proposition 1 (Partington and Bonnet, 2004) Let $\alpha=$ $\lim _{|s| \rightarrow \infty} Q_{\tau}(s) / Q_{0}(s)$.

i) If $|\alpha|<1$ then the poles of $G(s)$ of large modulii are asymptotic to the vertical line $\operatorname{Re}(s) \approx \log (|\alpha|) / \tau$ in the left half plane. The number of poles of $G(s)$ to the right of $\operatorname{Re}(s)=\log (|\alpha|) / \tau+\epsilon$ is finite for any $\epsilon>0$.

ii) If $|\alpha|>1$ then $G(s)$ has infinitely many unstable poles, asymptotic to the vertical line $\operatorname{Re}(s) \approx$ $\log (|\alpha|) / \tau$ in the right half plane.

As such, under the condition $|\alpha|<1$, there are at most a finite number of poles of $G(s)$ located in the right half plane. By scaling, this property extends to the half-plane to the right of any vertical line parallel to the imaginary axis, located to the right of the spectrum chain's asymptote. Note that for a retarded system, $\alpha=0$, and hence $G(s)$ always has a finite number of poles in the right half plane. In contrast, for a neutral system, if $|\alpha|>1$, this property will vanish. Note further that in general, $G(s)$ may contain multiple repeated poles, or equivalently, the quasipolynomial $Q_{0}(s)+Q_{\tau}(s) e^{-s \tau}$ may contain repeated zeros. In particular, in Boussaada and Niculescu (2016), it was shown that the algebraic multiplicity of a zero is bounded by the generic Polya/Szegö bound (Pólya and Szegö, 1972), which is the degree of the quasipolynomial. Here by the degree of a quasipolynomial, we mean the sum of the number of the involved polynomials and their degrees, less one; for the quasipolynomial $Q_{0}(s)+Q_{\tau}(s) e^{-s \tau}$, its degree is $\operatorname{deg}\left(Q_{0}\right)+\operatorname{deg}\left(Q_{\tau}\right)+1$.

The Fritz John condition (Bertsekas, 2016) is a firstorder necessary condition of optimality for constrained nonlinear programming problems whose objective function and constraints are differentiable, and the constraints may be equalities or inequalities. This class of problems can be stated as

$$
\begin{array}{ll} 
& \min f(x) \\
\text { s.t. } & g_{i}(x) \leq 0, \quad i \in \mathcal{I}_{1}=\{1,2, \cdots, m\}, \\
& h_{j}(x)=0, \quad j \in \mathcal{I}_{2}=\{m+1, m+2, \cdots, k\},
\end{array}
$$

where $f: \mathbb{R}^{n} \rightarrow \mathbb{R}, g_{i}: \mathbb{R}^{n} \rightarrow \mathbb{R}$ and $h_{j}: \mathbb{R}^{n} \rightarrow \mathbb{R}$ all have continuous first-order partial derivatives in $\mathbb{R}^{n}$.

Proposition 2 The Fritz John Condition (Bertsekas, 2016): If $x^{*}$ is an optimal solution of $f(x)$ in (2), then there exists a row vector $\lambda=\left[\lambda_{0}, \lambda_{1}, \lambda_{2}, \ldots, \lambda_{k}\right]$ such that:

$$
\begin{array}{r}
\lambda_{0} \nabla f\left(x^{*}\right)+\sum_{i \in \mathcal{I}_{1}} \lambda_{i} \nabla g_{i}\left(x^{*}\right)+\sum_{j \in \mathcal{I}_{2}} \lambda_{j} \nabla h_{j}\left(x^{*}\right)=0, \\
\sum_{i \in \mathcal{I}_{1}} \lambda_{i} g_{i}\left(x^{*}\right)=0, \\
\lambda_{i} \geq 0, i \in\{0\} \cup \mathcal{I}_{1}, \\
\lambda \neq 0,
\end{array}
$$

where $\nabla \theta(x)=\left[\partial \theta(x) / \partial x_{1}, \cdots, \partial \theta(x) / \partial x_{n}\right]^{\top}$ denotes the gradient of $\theta(x)$.

It is useful to note that the Fritz John condition is applicable to problems where the constraints may not satisfy certain regularity conditions, specifically when the gradient vectors $\nabla g_{i}\left(x^{*}\right)(i=1, \cdots, m)$ and $\nabla h_{j}^{*}$ $(j=m+1, \cdots, k)$ are linearly dependent, to which the seemingly better-known Karush-Kuhn-Tucker condition (Luenberger and Ye, 1984) fails to apply. On the other hand, when the gradient vectors are linearly independent, the Fritz John condition reduces to Karush-KuhnTucker criterion. For a comprehensive treatment of Fritz John condition, we refer to Mangasarian and Fromovitz (1967); Bertsekas (2016).

Finally, we shall also need the concept of concavity, quasi-concavity, and pseudo-concavity. 
Definition 3 (Boyd and Vandenberghe, 2004) A function $\vartheta: \mathbb{R}^{n} \rightarrow \mathbb{R}$ is said to be concave over a convex set $\mathcal{S} \subset \mathbb{R}^{n}$ if for any $x, y \in \mathcal{S}$,

$$
\vartheta((1-\gamma) x+\gamma y) \geq(1-\gamma) \vartheta(x)+\gamma \vartheta(y), \quad \gamma \in[0,1],
$$

and quasi-concave if

$$
\vartheta((1-\gamma) x+\gamma y) \geq \min \{\vartheta(x), \vartheta(y)\}, \quad \gamma \in[0,1] .
$$

Apparently, every concave function is quasi-concave. Concave and quasi-concave functions admit a unique maximum, which can be found using convex optimization methods.

Definition 4 (Boyd and Vandenberghe, 2004) A differentiable function $\vartheta: \mathbb{R}^{n} \rightarrow \mathbb{R}$ is said to be pseudo-concave over a convex set $\mathcal{S} \subset \mathbb{R}^{n}$ if for any $x, y \in \mathcal{S}$,

$$
\nabla \vartheta(x)^{\top}(y-x) \leq 0 \Rightarrow \vartheta(y)-\vartheta(x) \leq 0 .
$$

Pseudo-concave functions possess properties essentially similar to those of concave and quasi-concave functions. Likewise, a unique maximum exists and can be computed in a similar manner. In particular, for a univariate pseudo-concave optimization problem, i.e., a unimodal pseudo-concave problem, the optimal solution can be solved efficiently by gradient-based and bisection approaches.

\subsection{The delay margin and PID control}

Consider the feedback system depicted in Figure 1, in which $K(s)$ represents a finite-dimensional LTI controller, $P_{\tau}(s)$ denotes the plant containing a constant but uncertain delay $\tau$, with transfer function given by

$$
P_{\tau}(s)=P_{0}(s) e^{-\tau s}, \quad \tau \geq 0 .
$$

Here $P_{0}(s)$ represents a strictly proper finite dimensional delay-free plant. The controller $K(s)$ of interest is one

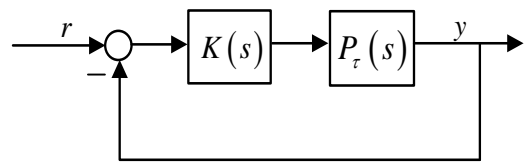

Figure 1. A tracking control system.

of PID controllers, i.e., $K(s)=K_{P I D}(s)$,

$$
K_{P I D}(s)=k_{p}+\frac{k_{i}}{s}+k_{d} s .
$$

The signals $r$ and $y$ represent the reference input and system output. Let

$$
\begin{gathered}
\tau\left(k_{p}, k_{i}, k_{d}\right)=\sup \left\{\mu \geq 0: K_{P I D}(s)\right. \text { stabilizes } \\
\left.P_{\tau}(s), \forall \tau \in[0, \mu)\right\} .
\end{gathered}
$$

Then $\tau\left(k_{p}, k_{i}, k_{d}\right)$ is the delay range within which the plant $P_{\tau}(s)$ can be robustly stabilized by the PID controller $K_{P I D}(s)$ with the fixed gains $\left(k_{p}, k_{i}, k_{d}\right)$. The delay margin of the system corresponds to the maximal range achievable by all PID controllers:

$$
\begin{gathered}
\bar{\tau}_{P I D}=\sup \left\{\tau\left(k_{p}, k_{i}, k_{d}\right): K_{P I D}(s)\right. \text { stabilizes } \\
\left.P_{\tau}(s), \forall \tau \in[0, \mu)\right\} .
\end{gathered}
$$

In this paper we consider the first-order delay plant

$$
P_{\tau}(s)=\frac{1}{s-p} e^{-\tau s}, \quad \tau \geq 0,
$$

where $p \geq 0$. For this class of plants, we have the following characterization of the delay margin, adapted from Ma and Chen (2019).

Proposition 5 Let $P_{\tau}(s)$ be given by (11). Then under the conditions $k_{p}>p, k_{i}>0$, and $\left|k_{d}\right|<1$,

$$
\tau\left(k_{p}, k_{i}, k_{d}\right)=\frac{\tan ^{-1} \frac{\omega_{0}}{p}}{\omega_{0}}+\frac{\tan ^{-1} \frac{k_{d} \omega_{0}-\frac{k_{i}}{\omega_{0}}}{k_{p}}}{\omega_{0}},
$$

where $\omega_{0}>0$ is given by

$$
\omega_{0}^{2}=\frac{\frac{k_{p}^{2}-2 k_{d} k_{i}-p^{2}}{1-k_{d}^{2}}+\sqrt{\left(\frac{k_{p}^{2}-2 k_{d} k_{i}-p^{2}}{1-k_{d}^{2}}\right)^{2}+4 \frac{k_{i}^{2}}{1-k_{d}^{2}}} .}{2} .
$$

Furthermore,

$$
\bar{\tau}_{P I D}=\frac{2}{p} .
$$

Let the open-loop transfer function of the delay-free system be defined by

$$
L_{0}(s)=P_{0}(s) K_{P I D}(s) .
$$

In the standard feedback design, the open-loop gain is to be shaped in such a way that $\left|L_{0}(j \omega)\right| \gg 1$ at low frequencies and $\left|L_{0}(j \omega)\right| \ll 1$ at high frequencies, thus necessitating the specification that $\left|L_{0}(0)\right|>1$ and $\left|L_{0}(\infty)\right|<1$. Here the former loopshaping specification is required to ensure such performance objective as attenuation of low-frequency disturbances, and the latter is imposed for reduction of high-frequency noises and to maintain stability robustness against high-frequency modeled dynamics. With the integral control in presence, the condition $\left|L_{0}(0)\right|>1$ is rendered moot whenever $k_{i}>0$. The requirement $\left|L_{0}(\infty)\right|<1$, on the other hand, coincides with the condition $\left|k_{d}\right|<1$. Note that for $K_{P I D}(s)$ to stabilize $P_{0}(s)$, additionally, it is necessary that $k_{p}>p$, and $k_{i}>0$. In summary, we assume in the sequel that $k_{p}>p, k_{i}>0$, and $\left|k_{d}\right|<1$. 


\section{Pole placement by PID control}

While maintaining the delay robustness stipulated in Proposition 5, it is desirable for the PID controller to also achieve desirable transient behavior. In this section we provide a design based on multiplicity-induced dominancy, which enforces the closed-loop poles as dominant multiple poles, so that the closed-loop response has a prescribed decay rate; in other words, the rightmost poles of the closed-loop system are placed at a same location. For this purpose, we first note that with the first-order plant $P_{\tau}(s)$ given by (11) and the PID controller given by $(10)$, the closed-loop quasipolynomial is found as

$$
\left\{\begin{aligned}
\Delta(s) & =Q_{0}(s)+Q_{\tau}(s) \mathrm{e}^{-s \tau} \\
Q_{0}(s) & =s^{2}-s p, \quad Q_{\tau}(s)=k_{d} s^{2}+k_{p} s+k_{i} .
\end{aligned}\right.
$$

Our first main result of this paper is stated as follows.

Theorem 6 Let $P_{\tau}(s)$ be given by (11)and $K_{P I D}(s)$ by (10). Then the following statements are true.

i) For arbitrary real parameters $k_{p}, k_{i}, k_{d}$ and arbitrary positive delay $\tau$, the multiplicity of any root of the quasipolynomial $\Delta(s)$ is less than or equal to 5 .

ii) The quasipolynomial $\Delta(s)$ admits a multiple real root at

$$
s_{ \pm}=\frac{\tau p-6 \pm \sqrt{\tau^{2} p^{2}+12}}{2 \tau}
$$

with algebraic multiplicity greater than 3 if and only if

$$
\left\{\begin{array}{l}
k_{d}=\frac{\left(4+2 \tau s_{ \pm}-\tau p\right) \mathrm{e}^{\tau s_{ \pm}}}{2} \\
k_{p}=-\frac{\left(\left(8 \tau+\tau^{2} s_{ \pm}\right) p-18-12 \tau s_{ \pm}\right) \mathrm{e}^{\tau s_{ \pm}}}{\tau} \\
k_{i}=\frac{\left(\left(\tau s_{ \pm}+3\right) \tau^{2} p^{2}-\left(12 \tau s_{ \pm}+60\right) \tau p+108+84 \tau s_{ \pm}\right) \mathrm{e}^{\tau s_{ \pm}}}{2 \tau^{2}}
\end{array}\right.
$$

iii) If (17) is satisfied, then under the condition $\tau<$ $\bar{\tau}_{P I D}=2 / p, s=s_{+}$is the rightmost root of the quasipolynomial $\Delta(s)$ with multiplicity equal to 4.

PROOF. i) The statement follows directly from the Pólya and Szegö bound (Pólya and Szegö, 1972), since the degree of the quasipolynomial $\Delta(s)$ is equal to 5 .

ii) For a root $s$ of the quasipolynomial $\Delta(s)$ given in (15), it follows that

$$
\mathrm{e}^{-\tau s}=\frac{-s^{2}+p s}{k_{d} s^{2}+k_{p} s+k_{i}} .
$$

The root $s$ has algebraic multiplicity greater than three if and only if in the ideal $\mathcal{I}_{4}=<\partial_{s} \Delta, \partial_{s}^{2} \Delta, \partial_{s}^{3} \Delta>$ generated by (18), the first-, second-, and third-order derivatives of $\Delta(s)$ are all zero at $s$. This results in three algebraic equations in 6 unknowns $k_{i}, k_{p}, k_{d}, \tau, p, s$ :

$$
\begin{aligned}
0= & \tau k_{d} s^{4}+\left(k_{p}-k_{d} p\right) \tau s^{3} \\
& +\left(\left(k_{i}-k_{p} p\right) \tau+k_{d} p+k_{p}\right) s^{2}+\left(2 k_{i}-\tau p k_{i}\right) s-p k_{i} \\
0= & -\tau^{2} k_{d} s^{4}+\left(\left(-k_{p}+k_{d} p\right) \tau^{2}+4 \tau k_{d}\right) s^{3} \\
& +\left(\left(k_{p} p-k_{i}\right) \tau^{2}+\left(-4 k_{d} p+2 k_{p}\right) \tau\right) s^{2} \\
& +\left(\tau^{2} p k_{i}+2 k_{p}+2 k_{d} p-2 \tau k_{p} p\right) s+2 k_{i}, \\
0= & { }^{3} k_{d} s^{4}+\left(\left(k_{p}-k_{d} p\right) \tau^{3}-6 \tau^{2} k_{d}\right) s^{3} \\
& +\left(\left(k_{i}-k_{p} p\right) \tau^{3}+\left(6 k_{d} p-3 k_{p}\right) \tau^{2}+6 \tau k_{d}\right) s^{2} \\
& +\left(-p k_{i} \tau^{3}+3 \tau^{2} k_{p} p-6 \tau k_{d} p\right) s
\end{aligned}
$$

By variable elimination, we obtain the following three sets of admissible solutions:

(1) $k_{i}=k_{p}=k_{d}=0$.

(2) $k_{i}=s=0$.

(3) $k_{p}=-2 \frac{\left(\left(\tau s_{ \pm}+8\right) \tau p-12 \tau s_{ \pm}-18\right) k_{d}}{\tau\left(\tau\left(2 s_{ \pm}-p\right)+4\right)}$,

$$
k_{i}=\frac{\left(\left(\tau s_{ \pm}+3\right) \tau^{2} p^{2}+\left(-12 \tau s_{ \pm}-60\right) \tau p+84 \tau s_{ \pm}+108\right) k_{d}}{\left(2 \tau s_{ \pm}+4-\tau p\right) \tau^{2}} .
$$

Evidently, the first two cases are not applicable and hence are discarded. In Case (3), substituting $k_{p}$ and $k_{i}$ into (18) yields the expression of $k_{d}$ in (17).

iii) The proof of the statement iii) is somewhat longwinding, and so for it to be more accessible, we divide the proof into three parts, each of which is preceded by a subheading.

iii-a) Establishing the multiplicity of $s_{+}$To determine the exact multiplicity of $s_{+}$, we consider the transformation $s \rightarrow(z / \tau)+s_{+}$and

$$
\hat{\Delta}(z)=\tau^{2} \Delta\left(\frac{z}{\tau}+s_{+}\right)
$$

Denote $\rho=\sqrt{\tau^{2} p^{2}+12}$. Under the condition $0<\tau<$ $\bar{\tau}_{P I D}=2 / p$, we have

$$
2 \sqrt{3}<\rho<4 \text {. }
$$


A direct calculation gives rise to

$$
\begin{aligned}
\hat{\Delta}(z)= & z^{2}+(\rho-6) z+(12-3 \rho) \\
& +\left(\frac{\rho-2}{2} z^{2}+(2 \rho-6) z+(3 \rho-12)\right) e^{-z}
\end{aligned}
$$

It follows that $s_{+}$is a root of $\Delta(s)$ with a certain algebraic multiplicity if and only if $z=0$ is a root of $\hat{\Delta}(z)$ with the same algebraic multiplicity. We then proceed to show that $\hat{\Delta}(z)$ has a root at the origin with algebraic multiplicity 4 . Toward this end, denote

$$
\begin{aligned}
& \hat{Q}_{0}(z)=z^{2}+(\rho-6) z+(12-3 \rho) \\
& \hat{Q}_{\tau}(z)=\frac{\rho-2}{2} z^{2}+(2 \rho-6) z+(3 \rho-12)
\end{aligned}
$$

and write

$$
\hat{\Delta}(z)=\hat{Q}_{0}(z)+\hat{Q}_{\tau}(z) e^{-z} .
$$

The quasipolynomial $\hat{\Delta}(z)$ has a root on the imaginary axis if and only if $\hat{\Delta}(j \omega)=0$. By a direct calculation, we find

$$
\left|\hat{Q}_{0}(j \omega)\right|^{2}-\left|\hat{Q}_{\tau}(j \omega)\right|^{2}=\left(1-\left(\frac{\rho-2}{2}\right)^{2}\right) \omega^{4} .
$$

Since $\rho<4$, it follows that for any $\omega \neq 0,\left|\hat{Q}_{0}(j \omega)\right|>$ $\left|\hat{Q}_{\tau}(j \omega)\right|$, and hence $\hat{\Delta}(j \omega) \neq 0$. To show that $\hat{\Delta}(0)=0$, we obtain the Taylor series of $\hat{\Delta}(z)$, given as

$$
\hat{\Delta}(z)=\frac{\rho}{24} z^{4}+o\left(|z|^{4}\right) .
$$

This enables us to conclude that $\hat{\Delta}(z)$ has a root at the origin with multiplicity 4 , and hence that $\Delta(s)$ has a root $s_{+}$with multiplicity 4 .

iii-b) Localizing the right half plane zeros of $\hat{\Delta}(z)$ We next show that $\hat{\Delta}(z)$ has no root in the open right half plane, i.e., for all $z$ such that $\operatorname{Re}(z)>0$. For this purpose, we first provide an auxiliary result, which is needed in the subsequent proof. This result is summarized in the following claim.

Claim Let $z_{0}=x_{0}+j \omega_{0}, x_{0}>0, \omega_{0} \geq 0$ be a root of $\hat{\Delta}(z)$. Then, $\omega_{0}<\pi$.

To establish this claim, we note that if $z_{0}$ is a root of $\hat{\Delta}(z)$, i.e., $\hat{\Delta}\left(z_{0}\right)=0$, then necessarily

$$
\left|\hat{Q}_{0}\left(x_{0}+j \omega_{0}\right)\right|^{2} \mathrm{e}^{2 x_{0}}=\left|\hat{Q}_{\tau}\left(x_{0}+j \omega_{0}\right)\right|^{2} .
$$

Consider then the function

$F(x, \omega)=\left|\hat{Q}_{\tau}(x+j \omega)\right|^{2}-(1+2 x)\left|\hat{Q}_{0}(x+j \omega)\right|^{2}$.
Since $\mathrm{e}^{2 x}>1+2 x$ for any $x>0$, we have $F\left(x_{0}, \omega_{0}\right)>0$, for any right half plane root $z_{0}=x_{0}+j \omega_{0}$ of $\hat{\Delta}(z)$. The idea then is to show that if $z_{0}=x_{0}+j \omega_{0}$ is a root of $\hat{\Delta}(z)$, with $x_{0}>0$ and $\omega_{0} \geq \pi$, then $F\left(x_{0}, \omega_{0}\right) \leq 0$, thus resulting a contradiction. Toward this end, we set $\Omega=\omega^{2}$ and find the explicit expression of $F(x, \omega)$ as

$$
\begin{aligned}
F(x, \sqrt{\Omega})= & \frac{\left(\rho^{2}-4 \rho-8 x\right)}{4} \Omega^{2} \\
& +\frac{x^{2}\left(\rho^{2}-12 \rho-8 x+48\right)}{2} \Omega \\
& -2 x^{5}+\frac{(\rho-8)(\rho-12)}{4} x^{4} \\
& +24(\rho-4) x^{3}+18(\rho-4)^{2} x^{2} .
\end{aligned}
$$

Fix $x>0$. The discriminant of the second-order polynomial in $\Omega$ is given by $D(x)=x^{2} \hat{D}(x)$, where

$$
\begin{aligned}
\hat{D}(x)= & 16\left(\rho^{2}-12\right) x^{2}-24(\rho-6)(\rho-4)^{2} x \\
& -18 \rho(\rho-4)^{3} .
\end{aligned}
$$

Evidently, for $\rho \in(2 \sqrt{3}, 4), D(x)>0$ for any $x>$ 0 . This means that the polynomial $F(x, \sqrt{\Omega})$ has two real roots, which are given at the top of the next page. Here $\Omega_{+}(x)$ is the larger of the two roots. Accordingly, $F(x, \sqrt{\Omega})$ can be factorized as

$$
F(x, \sqrt{\Omega})=\frac{\left(\rho^{2}-4 \rho-8 x\right)}{4}\left(\Omega-\Omega_{-}(x)\right)\left(\Omega-\Omega_{+}(x)\right) .
$$

By judiciously setting $\rho=2 \sqrt{3}$ or $\rho=4$, we may establish the bound $\Omega_{+}(x) \leq \Gamma(x)$, where

$$
\begin{aligned}
\Gamma(x)= & -x^{2}-3 \sqrt{3} x+\frac{15 x}{2} \\
& +\sqrt{(-228 x+468) \sqrt{3}+4 x^{2}+396 x-810 .} .
\end{aligned}
$$

Taking the derivative of $\Gamma(x)$ gives rise to

$$
\begin{aligned}
\Gamma^{\prime}(x)= & -2 x-3 \sqrt{3}+\frac{15}{2} \\
& +\frac{-228 \sqrt{3}+8 x+396}{2 \sqrt{(-228 x+468) \sqrt{3}+4 x^{2}+396 x-810}},
\end{aligned}
$$

which yields the unique positive stationary point $x^{*} \approx 2.139487015$. It thus follows that $\Omega_{+}(x) \leq$ $\Gamma\left(x^{*}\right) \approx 4.961051386<\pi^{2}$. From (24), it is clear that for $F(x, \omega)>0$ for any $x>0$, it is necessary that $\omega<\pi$. As such, for $F\left(x_{0}, \omega_{0}\right)>0$ with $x_{0}>0, \omega_{0} \geq 0$, it is necessary that $\omega_{0}<\pi$, whereas the condition $F\left(x_{0}, \omega_{0}\right)>0$ in turn is necessary for (23) to hold. We have thus established the claim. 


$$
\Omega_{ \pm}(x)=\frac{\left(-8 x^{2}+\left(\rho^{2}-12 \rho+48\right) x \pm 2 \sqrt{\left(16 \rho^{2}-192\right) x^{2}-24(\rho-6)(\rho-4)^{2} x-18 \rho(\rho-4)^{3}}\right) x}{-\rho^{2}+4 \rho+8 x} .
$$

iii-c) Establishing the dominance of $s_{+}$To complete the proof, we employ the Fredholm integral representation of $\hat{\Delta}(z)$ (Kress, 2014). By a direct calculation, one can show that $\hat{\Delta}(z)$ admits the Fredholm integral form

$$
\hat{\Delta}(z)=\int_{0}^{1} \mathcal{K}(t, z) q(t) \mathrm{d} t,
$$

where the kernel function $\mathcal{K}(t, z)$ and $q(t)$ are given by

$$
\begin{aligned}
& \mathcal{K}(t, z)=\frac{z^{4} e^{-t z}}{2}, \\
& q(t)=t(1-t)((\delta-4) t+2),
\end{aligned}
$$

respectively. Note that for $\rho \in(2 \sqrt{3}, 4), q(t)$ is strictly positive over the interval $(0,1)$. Clearly, the Fredholm integral representation (25) can be simplified to

$$
\hat{\Delta}(z)=\frac{z^{4}}{2} \int_{0}^{1} q(t) \mathrm{e}^{-z t} \mathrm{~d} t .
$$

Assume that $\hat{\Delta}\left(x_{0}+j \omega_{0}\right)=0$ for some $x_{0}>0$ and $\omega_{0} \geq 0$. Then necessarily,

$$
\left\{\begin{array}{l}
\int_{0}^{1} q(t) \mathrm{e}^{-x_{0} t} \cos \left(\omega_{0} t\right) \mathrm{d} t=0, \\
\int_{0}^{1} q(t) \mathrm{e}^{-x_{0} t} \sin \left(\omega_{0} t\right) \mathrm{d} t=0 .
\end{array}\right.
$$

Since $0<\omega_{0}<\pi, \sin \left(\omega_{0} t\right)>0$ for $t \in(0,1)$. Likewise, $q(t)>0$ for $t \in(0,1)$. Consequently,

$$
\int_{0}^{1} q(t) \mathrm{e}^{-x_{0} t} \sin \left(\omega_{0} t\right) \mathrm{d} t>0
$$

which contradicts to $(26)$. This proves that $\hat{\Delta}(z) \neq 0$ in the right half plane and hence establishes the dominance of $s_{+}$, thus completing the proof.

Theorem 6 indicates that for any $\tau<\bar{\tau}_{P I D}=2 / p$, by enforcing the PID control gains as in (17), we may always place the closed-loop dominant poles at $s_{+}$given in (16), which is a multiple negative real pole and hence guarantees that the closed-loop system is stable with a specified degree of stability. Note that for delay robustness (with respect to variation in delay) it is natural to impose the condition $\tau<\bar{\tau}_{P I D}=2 / p$. Note also that implicit in the selection of the PID gains in accordance with (17), the derivative control gain $k_{d}$ satisfies the condition $\left|k_{d}\right|<1$

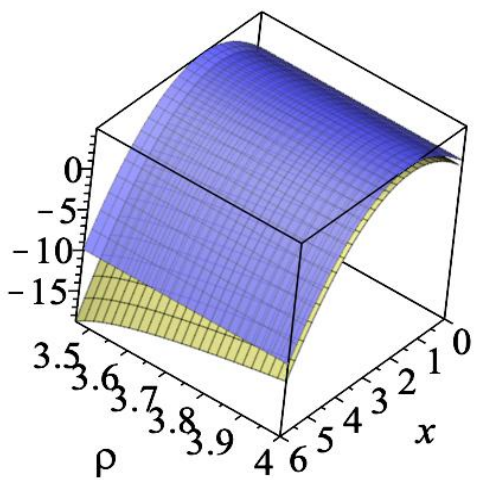

Figure 2. 3D-plot of $\Omega_{+}(x)$ (yellow) and $\Gamma(x)$ (blue).

(cf. Theorem 9). As noted at the end of Section 2, this condition coincides with the open-loop roll-off requirement. Inadvertently, it also guarantees that only a finite number of roots of $\Delta(s)$ exist in the shifted right-half complex plane $\mathbb{C}_{s_{+}}^{+}=\left\{s \in \mathbb{C}\right.$, s.t. $\left.\operatorname{Re}(s)>s_{+}\right\}$. To see this point, we note that

$$
\alpha=\lim _{|s| \rightarrow \infty} Q_{\tau}(s) / Q_{0}(s)=k_{d} .
$$

In light of Proposition 1, whenever $\left|k_{d}\right|<1, \Delta(s)$ has only a finite number of roots to the right of the line $\operatorname{Re}(s)=\log \left|k_{d}\right| / \tau$, whereas

$$
\begin{aligned}
\log \left|k_{d}\right| & =\log \left(\left(\frac{\sqrt{\tau^{2} p^{2}+12}}{2}-1\right) \mathrm{e}^{\tau s_{+}}\right) \\
& =\tau s_{+}-\log 2+\log \left(\sqrt{\tau^{2} p^{2}+12}-2\right) .
\end{aligned}
$$

Under the condition $\tau<\bar{\tau}_{P I D}=2 / p$, we have $\sqrt{\tau^{2} p^{2}+12}<4$, and hence $\log \left|k_{d}\right| / \tau<s_{+}$. This allows us to conclude that $\Delta(s)$ has only a finite number of roots in $\mathbb{C}_{s_{+}}^{+}$. Theorem 6 demonstrates further that $\Delta(s)$ in fact has no root in this region. Note additionally that the root $s_{-}$is of no interest, since it leads to $k_{d}<-1$; in other words, the resulting PID controller can not stabilize $P_{0}(s)$.

A number of remarks are now in order.

Remark 7 To further illustrate the proof of Theorem 6 , we plot $\Omega_{+}(x)$ and $\Gamma(x)$ in Figure 2, for $\rho \in(2 \sqrt{3}, 4)$ and a range of positive real $x$. One can see that within this range, $\Gamma(x)$ serves as an upper bound of $\Omega_{+}(x)$ independent of $\rho$, which does not exceed the value of 5 . Figure 3 shows that $\Gamma(x)$ has a unique maximum, and outside a certain range of $x$, it assumes negative values. 


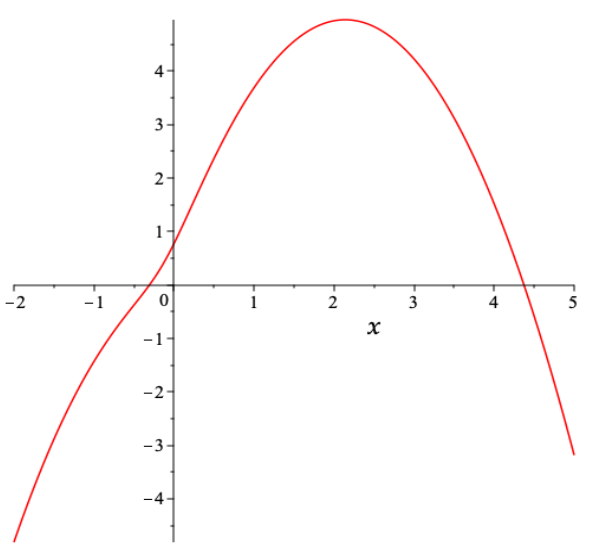

Figure 3. The function $\Gamma(x)$.

Remark 8 The MID scheme can be employed for the analytic design and tuning of PI controllers, with dominating multiple closed-loop poles as well. Indeed, in the case of a PI controller, the closed-loop characteristic function is given by

$$
\Delta(s)=s^{2}-s p+\left(k_{p} s+k_{i}\right) \mathrm{e}^{-s \tau} .
$$

Define analogously the delay margin achievable by PI controllers, and denote it by $\bar{\tau}_{P I}$. Then from $M a$ and Chen (2019), it is known that for the first-order plant (11), $\bar{\tau}_{P I}=1 / p$. By selecting

$$
s_{+}=\frac{\tau p-4+\sqrt{\tau^{2} p^{2}+8}}{2 \tau}
$$

and

$$
\begin{aligned}
& k_{p}=\frac{\left(\sqrt{\tau^{2} p^{2}+8}-2\right) \mathrm{e}^{\tau s_{+}}}{\tau}, \\
& k_{i}=\frac{\left((10-p \tau) \sqrt{\tau^{2} p^{2}+8}+2 \tau p-\tau^{2} p^{2}-28\right) \mathrm{e}^{\tau s_{+}}}{2 \tau^{2}},
\end{aligned}
$$

one can show analogously that under the condition $\tau<$ $\bar{\tau}_{P I}=1 / p, s=s_{+}$is the dominating root of $\Delta(s)$ with multiplicity 3.

We end our discussions by commenting briefly on the analytical MID tuning rule specified by (17). It is worth noting that the classical Ziegler-Nichols tuning rules, widely used for tuning PID controllers for stable processes, may have serious drawbacks and are not readily applicable to unstable plants (Åström and Hägglund, 2004); for example, the Ziegler-Nichols' first and second methods rely on open-loop step and sinusoidal responses, which for open-loop unstable systems are difficult to measure. For this reason, PID controller tuning rules for unstable processes have been developed by focusing on the direct synthesis of the closed-loop response or the numerical optimization of a certain closed-loop performance objective, resulting in a wide variety of tuning rules that differ widely from each other (see, e.g., (O'Dwyer, 2013; Thomas and Ganesan, 2020) and the references therein). Unlike these earlier rules, the MID tuning rule presented herein adds an analytical tool to the available repertoire, which, as shown in the sequel, possesses desirable robustness properties.

\section{Robustness of MID design}

In this section we show that while tuned to the delay parameter and the value of the plant unstable pole, the PID controller (17) by the MID design can nevertheless render desirable robustness properties. In this vein, we first show that the MID design ensures a certain amount of robustness against uncertain delay, which in the limit approaches to its maximum, namely, the delay margin. This result is summarized in the following theorem.

Theorem 9 Let $\left(k_{d}, k_{p}, k_{i}\right)$ be given in (17). Then the following statements are true.

i) For any $\tau<\bar{\tau}_{P I D}=2 / p$,

$$
\left\{\begin{array}{r}
0<k_{d}<1 \\
k_{p}>p \\
k_{i}>0
\end{array}\right.
$$

The PID controller $K_{P I D}(s)$ stabilizes $P_{\tau}(s)$ for all $\tau \in[0, \bar{\tau})$, where

$$
\bar{\tau}=\frac{\tan ^{-1} \frac{\omega_{0}}{p}}{\omega_{0}}+\frac{\tan ^{-1} \frac{k_{d} \omega_{0}-\frac{k_{i}}{\omega_{0}}}{k_{p}}}{\omega_{0}}
$$

with $\omega_{0}$ given by

$$
\omega_{0}^{2}=\frac{\frac{k_{p}^{2}-2 k_{d} k_{i}-p^{2}}{1-k_{d}^{2}}+\sqrt{\left(\frac{k_{p}^{2}-2 k_{d} k_{i}-p^{2}}{1-k_{d}^{2}}\right)^{2}+4 \frac{k_{i}^{2}}{1-k_{d}^{2}}}}{2} .
$$

ii) When $\tau \rightarrow \bar{\tau}_{P I D}=2 / p$,

$$
\left\{\begin{array}{l}
k_{d} \rightarrow 1 \\
k_{p} \rightarrow p \\
k_{i} \rightarrow 0
\end{array}\right.
$$

and $s_{+} \rightarrow 0$. The PID controller $K_{P I D}(s)$ stabilizes $P_{\tau}(s)$ for all $\tau \in\left[0, \bar{\tau}_{P I D}\right)$.

PROOF. To prove $i$ ), we first note that

$$
\tau s_{+}=\frac{\tau p-6+\sqrt{(\tau p)^{2}+12}}{2} .
$$


For brevity, denote $\tau p=\chi$. Then for any $\tau<2 / p$, $\chi<2$. Accordingly,

$$
\tau s_{+}=\frac{\chi-6+\sqrt{\chi^{2}+12}}{2}
$$

which allows us to rewrite (17) as

$$
\left\{\begin{array}{l}
k_{d}=f(\chi) \mathrm{e}^{\tau s_{+}}, \\
k_{p}=\frac{g(\chi)}{\tau} \mathrm{e}^{\tau s_{+}}, \\
k_{i}=\frac{h(\chi)}{\tau^{2}} \mathrm{e}^{\tau s_{+}} .
\end{array}\right.
$$

where

$$
\left\{\begin{aligned}
f(\chi)= & \frac{\sqrt{\chi^{2}+12}}{2}-1 \\
g(\chi)= & \left(6-\frac{\chi}{2}\right) \sqrt{\chi^{2}+12}-\frac{\chi^{2}}{2}+\chi-18 \\
h(\chi)= & \left(\frac{\chi^{2}}{2}-6 \chi+42\right) \sqrt{\chi^{2}+12} \\
& +\frac{\chi^{3}}{2}-6 \chi^{2}+18 \chi-144
\end{aligned}\right.
$$

Obviously, $f(\chi)>0$. For any $\chi<2$, we have $f(\chi)<1$. Since $\mathrm{e}^{\tau s_{+}}<1, k_{d}=f(\chi) \mathrm{e}^{\tau s_{+}}<1$. Consider next the function

$$
\hat{g}(\chi)=g(\chi) \mathrm{e}^{g_{1}(\chi)}-\chi,
$$

where

$$
g_{1}(\chi)=\frac{\chi-6+\sqrt{\chi^{2}+12}}{2} .
$$

We prove below that $\hat{g}(\chi)$ is monotonically increasing on $[0,2)$. For this purpose we consider its first- and secondorder derivatives

$$
\hat{g}^{\prime}(\chi)=\frac{(\chi-5) \sqrt{\chi^{2}+12}-\chi^{2}+5 \chi-8}{2} \mathrm{e}^{g_{1}(\chi)}-1
$$

and

$\hat{g}^{\prime \prime}(\chi)=\left(\frac{-\chi^{2}+3 \chi-3}{2}+\frac{\chi^{3}-3 \chi^{2}+9 \chi-18}{2 \sqrt{\chi^{2}+12}}\right) \mathrm{e}^{g_{1}(\chi)}$.

One can easily show that $\hat{g}^{\prime \prime}(\chi)$ is negative since both the polynomials $g_{2}(\chi)=-\chi^{2}+3 \chi-3$ and $g_{3}(\chi)=$ $\chi^{3}-3 \chi^{2}+9 \chi-18$ are negative on the interval $[0,2)$. In other words, $\hat{g}^{\prime}(\chi)$ is a decreasing function over the interval $[0,2)$, which in turn means that

$$
\inf _{\chi \in[0,2)} \hat{g}^{\prime}(\chi)=\hat{g}^{\prime}(2)=0 .
$$

As such, on $[0,2), \hat{g}^{\prime}(\chi)>0$, which implies that $\hat{g}(\chi)$ is monotonically increasing, and for $0<\chi<2$,

$$
\hat{g}(\chi)=g(\chi) \mathrm{e}^{g_{1}(\chi)}-\chi>\hat{g}(0)>0,
$$

or equivalently

$$
g(\chi) \mathrm{e}^{g_{1}(\chi)}>\chi
$$

Substituting now $\chi=\tau p$, we have

$$
k_{p}=\frac{g(\chi)}{\tau} e^{\tau s_{+}}>p .
$$

Finally, consider the parameter $k_{i}$. We show that $h(\chi)>$ 0 . Toward this end, we first find

$$
h^{\prime}(\chi)=3 \frac{(\chi-2)\left((\chi-6)\left(\sqrt{\chi^{2}+12}+\chi\right)+24\right)}{2 \sqrt{\chi^{2}+12}} .
$$

For $\chi<2$, it is immediate that

$$
(\chi-6)\left(\sqrt{\chi^{2}+12}+\chi\right)+24<0,
$$

and hence $h^{\prime}(\chi)<0$. In other words, $h(\chi)$ is monotonically decreasing on $[0,2)$, and

$$
\inf _{\chi \in[0,2)} \hat{h}(\chi)=\hat{h}(2)=0 .
$$

This proves that $k_{i}>0$. Under the condition (27), it follows from Proposition 5 that $P_{\tau}(s)$ can be stabilized robustly by $K_{P I D}(s)$ for all $\tau \in[0, \bar{\tau})$, where $\bar{\tau}$ is given by (28). This completes the proof for the statement $i$ ). To prove the statement $i i$ ), it suffices to note that when $\tau \rightarrow \bar{\tau}_{P I D}, s_{+} \rightarrow 0$.

Remark 10 From (16), a direct calculation shows that

$$
\frac{d s_{+}}{d \tau}=\frac{3}{\tau^{2}}\left(1-\frac{2}{\sqrt{\tau^{2} p^{2}+12}}\right) .
$$

Hence, for any $\tau<2 / p, d s_{+} / d \tau>0$, that is, $s_{+}$increases monotonically with $\tau$, thus demonstrating a tradeoff between the delay $\tau$ selected and the relative stability of the closed-loop system. It is intuitively plausible to contend that an inherent tradeoff may exist as well between the dominant root $s_{+}$and the delay margin $\bar{\tau}$. The relationship between these two quantities, however, appears highly complex to prevent from an analytical characterization, whilst examples can be constructed to show that a monotone relationship does exist (cf. Section 6). Interestingly, the example presented in Section 6 also shows that not only does the MID design guarantee a certain amount of delay robustness, but it in fact yields a delay margin, surprisingly, greater than the value of $\tau$ selected. 
It is of interest to analyze the robustness of the MID design to the variation in the plant unstable pole. For this purpose, we examine the uncertain plant

$$
\hat{P}_{\tau}(s)=\frac{1}{s-\hat{p}} e^{-\tau s}, \quad \tau \geq 0,
$$

with an uncertain pole $\hat{p} \in[p-\nu, p+\nu]$, for some prescribed $\nu>0$. Define analogously

$$
\Delta(s, \hat{p})=s^{2}-s \hat{p}+\left(k_{d} s^{2}+k_{p} s+k_{i}\right) e^{-s \tau} .
$$

Under the condition $\tau<\bar{\tau}_{P I D}=2 / p$, let $\left(k_{p}, k_{i}, k_{d}\right)$ be given by the MID design (17) with the nominal value $p$ of the plant pole. We first analyze the variation of the dominant pole $s_{+}$under the perturbation $\hat{p}-p$, which can be formulated as an eigenvalue perturbation problem. Specifically, by exploiting the results of (Chen et al., 2017), we assert that the multiple pole $s_{+}$splits into four branches, which in the neighborhood of $s_{+}$can be described asymptotically as

$$
\begin{aligned}
s_{+}^{(k)}=s_{+}+(-4 ! & \left.\frac{\left.\frac{\partial \Delta\left(s_{+}, \hat{p}\right)}{\partial \hat{p}}\right|_{\hat{p}=p}}{\left.\frac{\partial^{4} \Delta(s, p)}{\partial s^{4}}\right|_{s=s_{+}}}\right)^{1 / 4}(\hat{p}-p)^{1 / 4} \\
& +o\left((\hat{p}-p)^{1 / 4}\right), \quad k=1,2,3,4 .
\end{aligned}
$$

Here for a complex number $z$, we denote its $m$-th roots by

$$
z^{1 / m}=|z|^{1 / m} e^{j(2(k-1) \pi+\varangle z) / m}, \quad k=1,2, \cdots, m .
$$

It is evident that

$$
\left.\frac{\partial \Delta\left(s_{+}, \hat{p}\right)}{\partial \hat{p}}\right|_{\hat{p}=p}=-s_{+} .
$$

Furthermore, since $\Delta(s, p)=\Delta(s)=\hat{\Delta}\left(\tau\left(s-s_{+}\right)\right) / \tau^{2}$, it follows from (22) that

$$
\left.\frac{\partial^{4} \Delta(s, p)}{\partial s^{4}}\right|_{s=s_{+}}=\rho \tau^{2} .
$$

Consequently, we find that for $k=1,2,3,4$,

$s_{+}^{(k)}=s_{+}+\left(\frac{4 !}{\rho \tau^{2}}\right)^{1 / 4}\left(s_{+}\right)^{1 / 4}(\hat{p}-p)^{1 / 4}+o\left((\hat{p}-p)^{1 / 4}\right)$.

Note that for $\hat{p}>p$, since $s_{+}<0$, the dominant pole $s_{+}$ will split into four branches with asymptotic departure angles as $\pi / 4,3 \pi / 4,5 \pi / 4$, and $7 \pi / 4$, respectively. As $\hat{p}$ increases, two of the four poles at $s_{+}$will emerge as a pair of dominate conjugate complex poles with asymptotes oriented at the angles of $\pi / 4$ and $-\pi / 4$.

\section{Tradeoff between delay robustness and} steady-state tracking

With its integral control action, PID control generally performs well with set-point reference tracking. On the other hand, Theorem 9 suggests that to gain a maximal delay margin, the integral control effort must be reduced, thus demonstrating a tradeoff between steadystate tracking and delay robustness, which more generally can be attributed as a manifestation of the tradeoff between control performance and system robustness. In this section, we characterize this tradeoff in a precise manner.

For the feedback configuration shown in Figure 1, the tracking error signal is defined by

$$
e(t)=y(t)-r(t) .
$$

Let the Laplace transforms of the reference input $r(t)$ and the tracking error signal $e(t)$ be denoted as $\hat{r}(s)$ and $\hat{e}(s)$. It follows that

$$
\hat{e}(s)=\frac{1}{1+L_{0}(s) e^{-\tau s}} \hat{r}(s),
$$

where $L_{0}(s)$ is given by (14). Provided that the closedloop system is stable, the steady-state tracking error is determined as

$$
e(\infty)=\lim _{t \rightarrow \infty} e(t)=\lim _{s \rightarrow 0} s \hat{e}(s) .
$$

Evidently, with a step reference signal, perfect steadystate tracking is achieved whenever the integral control is applied, that is, the steady-state tracking error is zero.

Consider then the more demanding task of tracking a ramp reference signal:

$$
r(t)= \begin{cases}t & t \geq 0 \\ 0 & t<0 .\end{cases}
$$

In this case, the integral control is clearly necessary; otherwise, the asymptotic tracking error would be infinitely large. For the first-order plant given in (11), the absolute tracking error becomes

$$
|e(\infty)|=\lim _{s \rightarrow 0}\left|\frac{s}{1+L_{0}(s) e^{-\tau s}} \hat{r}(s)\right|=\frac{p}{k_{i}},
$$

whenever the closed-loop system is stable. In other words, to ensure a small $|e(\infty)|$, it is necessary to make $k_{i}$ large. To meet this requirement, we enforce the specification given in

Assumption $11 k_{i} \geq \sigma>0$. 
Under this assumption, define

$$
\Xi(\sigma)=\left\{\left(k_{p}, k_{i}, k_{d}\right): k_{p}>p,\left|k_{d}\right|<1, k_{i} \geq \sigma\right\},
$$

and

$$
\bar{\tau}_{P I D}(\sigma)=\sup \left\{\tau\left(k_{p}, k_{i}, k_{d}\right):\left(k_{p}, k_{i}, k_{d}\right) \in \Xi(\sigma)\right\}
$$

Then $\bar{\tau}_{P I D}(\sigma)$ defines the maximal delay margin achievable contingent upon the tracking performance desired. Note that $\bar{\tau}_{P I D}(0)=\bar{\tau}_{P I D}$. The following result characterizes this delay margin, and henceforth the tradeoff in achieving delay robustness and the tracking performance.

Theorem 12 Let $P_{\tau}(s)$ be given by (11). Then the following statements hold:

i)

$$
\bar{\tau}_{P I D}(\sigma)=\sup _{p<k_{p}<\sqrt{p^{2}+2 \sigma}} \hat{\tau}\left(k_{p}\right)
$$

where

$$
\hat{\tau}\left(k_{p}\right)=\lim _{k_{d} \rightarrow 1} \tau\left(k_{p}, \sigma, k_{d}\right),
$$

with $\tau\left(k_{p}, k_{i}, k_{d}\right)=\bar{\tau}$ in $(28)$, and $\omega_{0}$ given by

$$
\omega_{0}=\frac{\sigma}{\sqrt{p^{2}-k_{p}^{2}+2 \sigma}}
$$

ii) $\hat{\tau}\left(k_{p}\right)$ is pseudo-concave for $p<k_{p}<\sqrt{p^{2}+2 \sigma}$.

iii) $\bar{\tau}_{P I D}(\sigma)$ is strictly decreasing with $\sigma \geq 0$.

iv)

$$
\bar{\tau}_{P I D}(\sigma) \leq\left(\frac{\tan ^{-1} \frac{\sqrt{\frac{\sigma}{2}}}{p}}{\frac{\sqrt{\frac{\sigma}{2}}}{p}}\right) \frac{2}{p}
$$

It is clear from i) that for a first-order unstable system, the optimal derivative control coefficient $k_{d}$ lies (asymptotically) on the boundary of its allowable range, i.e., $k_{d}=1$, pointing to the fact that the derivative control has a positive effect to compensate for the time delay. On the contrary, the delay margin $\bar{\tau}_{P I D}(\sigma)$ is achieved when the integral control gain is minimal, i.e., $k_{i}=\sigma$, indicating that the integral control contributes no effect to enlarge the delay margin. The pseudo-concavity of $\hat{\tau}\left(k_{p}\right)$ reveals that the exact determination of the delay margin amounts to solving a pseudo-concave unimodal problem, which can be solved using convex optimization or gradient-based methods. The monotonicity property established iii) demonstrates the tradeoff between delay robustness and tracking accuracy, which can be further observed by the explicit a priori bound stated iv).
We now provide the proof of Theorem 12. Similarly, we also provide subheadings as a guide to the proof.

PROOF. i-a) Reformulation as a nonlinear programming problem We begin by recognizing the fact that the set $\Xi(\sigma)$ parameterizes the PID coefficients such that the closed-loop system is stable, and the open-loop gain satisfies the roll-off condition $\left|L_{0}(\infty)\right|<1$, together with the steady-state tracking specification prescribed by Assumption 11. Under these constraints, we seek to maximize $\tau\left(k_{p}, k_{i}, k_{d}\right)$. Our first step is to recast the maximization problem as one of constrained nonlinear programming:

$$
\begin{aligned}
& \min \quad f\left(k_{p}, k_{i}, k_{d}, \omega_{0}\right) \\
& \text { s.t. } g_{1}\left(k_{p}, k_{i}, k_{d}, \omega_{0}\right)=p-k_{p} \leq 0 \\
& g_{2}\left(k_{p}, k_{i}, k_{d}, \omega_{0}\right)=k_{d}-1 \leq 0 \\
& g_{3}\left(k_{p}, k_{i}, k_{d}, \omega_{0}\right)=-k_{d}-1 \leq 0 \\
& g_{4}\left(k_{p}, k_{i}, k_{d}, \omega_{0}\right)=\sigma-k_{i} \leq 0 \\
& h\left(k_{p}, k_{i}, k_{d}, \omega_{0}\right)=\left(1-k_{d}^{2}\right) \omega_{0}^{4} \\
&+\left(p^{2}-k_{p}^{2}+2 k_{d} k_{i}\right) \omega_{0}^{2}-k_{i}^{2}=0 .
\end{aligned}
$$

where

$$
f\left(k_{p}, k_{i}, k_{d}, \omega_{0}\right)=-\frac{\tan ^{-1} \frac{\omega_{0}}{p}}{\omega_{0}}-\frac{\tan ^{-1} \frac{k_{d} \omega_{0}-\frac{k_{i}}{\omega_{0}}}{k_{p}}}{\omega_{0}} .
$$

Note that in this formulation, the first four inequality constraints represent the set $\Xi(\sigma)$, where the strict inequalities are modified to enable the equivalence between the infimum and minimum. The last equality constraint characterizes the crossover frequency $\omega_{0}$, i.e., the solution to $h\left(k_{p}, k_{i}, k_{d}, \omega_{0}\right)=0$. It is clear that

$$
\begin{aligned}
\bar{\tau}_{P I D}(\sigma) & =\sup _{\left(k_{p}, k_{i}, k_{d}\right) \in \Xi(\sigma)} \tau\left(k_{p}, k_{i}, k_{d}\right) \\
& =\min _{g_{1}, g_{2}, g_{3}, g_{4}, h} f\left(k_{p}, k_{i}, k_{d}, \omega_{0}\right) .
\end{aligned}
$$

i-b) Establishing the necessity of $k_{i}=\sigma, k_{d}=$ 1 for optimality Note that since the equation $h\left(k_{p}, k_{i}, k_{d}, \omega_{0}\right)=0$ admits a unique solution given by $(29)$, the objective function $f\left(k_{p}, k_{i}, k_{d}, \omega_{0}\right)$ is differentiable. This allows us to invoke the Fritz John condition, which gives rise the first-order conditions

$$
\begin{aligned}
& \lambda_{0} \nabla f\left(k_{p}^{*}, k_{i}^{*}, k_{d}^{*}, \omega_{0}^{*}\right)+\sum_{i=1}^{4} \lambda_{i} \nabla g_{i}\left(k_{p}^{*}, k_{i}^{*}, k_{d}^{*}, \omega_{0}^{*}\right) \\
& +\lambda_{5} \nabla h\left(k_{p}^{*}, k_{i}^{*}, k_{d}^{*}, \omega_{0}^{*}\right)=0, \\
& \sum_{i=1}^{4} \lambda_{i} g_{i}\left(k_{p}^{*}, k_{i}^{*}, k_{d}^{*}, \omega_{0}^{*}\right)=0 \\
& \lambda_{i} \geq 0, i \in\{0, \ldots, 4\}, \\
& \lambda=\left[\lambda_{0}, \lambda_{1}, \lambda_{2}, \lambda_{3}, \lambda_{4}, \lambda_{5}\right] \neq 0 \text {. }
\end{aligned}
$$


We then examine the partial derivatives of $f\left(k_{p}, k_{i}, k_{d}, \omega_{0}\right)$, $h\left(k_{p}, k_{i}, k_{d}, \omega_{0}\right)$ and $g_{i}\left(k_{p}, k_{i}, k_{d}, \omega_{0}\right), i=1, \ldots, 4$ with respect to $k_{d}, k_{i}$ and $k_{p}$, which results in the equations

$$
\begin{array}{cc}
\frac{\partial}{\partial k_{d}}: & \lambda_{0} \frac{1}{1+\Pi^{2}} \frac{1}{k_{p}^{*}}-\lambda_{2}+\lambda_{3}+2 \lambda_{5}\left(k_{d}^{*} \omega_{0}^{* 2}-k_{i}^{*}\right) \omega_{0}^{* 2}=0, \\
\frac{\partial}{\partial k_{i}}: & \lambda_{0} \frac{1}{1+\Pi^{2}} \frac{1}{k_{p}^{*}}-\lambda_{4} \omega_{0}^{* 2}+2 \lambda_{5}\left(k_{d}^{*} \omega_{0}^{* 2}-k_{i}^{*}\right) \omega_{0}^{* 2}=0, \\
\frac{\partial}{\partial k_{p}}: & \lambda_{0} \frac{1}{1+\Pi^{2}}\left(k_{d}^{*}-\frac{k_{i}^{*}}{\omega_{0}^{* 2}}\right) \frac{1}{k_{p}^{* 2}}-\lambda_{1}-2 \lambda_{5} k_{p}^{*} \omega_{0}^{* 2}=0,
\end{array}
$$

where $\Pi=\left(k_{d}^{*} \omega_{0}^{*}-k_{i}^{*} / \omega_{0}^{*}\right) / k_{p}^{*}$. In light of the condition $\sum_{i=1}^{4} \lambda_{i} g_{i}\left(k_{p}^{*}, k_{i}^{*}, k_{d}^{*}, \omega_{0}^{*}\right)=0$, it follows that $g_{i}\left(k_{p}^{*}, k_{i}^{*}, k_{d}^{*}, \omega_{0}^{*}\right)=0$ whenever $\lambda_{i}>0$, which means that the constraint $g_{i} \leq 0$ is active. Note that the equations (38) and (39) give rise to

$$
-\lambda_{2}+\lambda_{3}=-\lambda_{4} \omega_{0}^{* 2}
$$

Since $g_{2}\left(k_{p}^{*}, k_{i}^{*}, k_{d}^{*}, \omega_{0}^{*}\right)=0$ and $g_{3}\left(k_{p}^{*}, k_{i}^{*}, k_{d}^{*}, \omega_{0}^{*}\right)=0$ are mutually exclusive, $\lambda_{2}$ and $\lambda_{3}$ cannot be both positive. Hence for (41) to hold, either $\lambda_{2}=\lambda_{3}=\lambda_{4}=0$ or $\lambda_{3}=$ $0, \lambda_{2}=\lambda_{4} \omega_{0}^{* 2}$. Consider first the case $\lambda_{2}=\lambda_{3}=\lambda_{4}=0$. The discussion of this case can be further divided into two parts.

1) $k_{d}^{*} \omega_{0}^{* 2}-k_{i}^{*}<0$. If $\lambda_{0}=0$, then $\lambda_{5}=0$ by (38). Since $\lambda \neq 0$, we have $\lambda_{1} \neq 0$. This, however, contradicts to (40). Hence, we assert that $\lambda_{0}>0$. Under this condition, it follows from (38) that $\lambda_{5}>0$. Nevertheless, that both $\lambda_{0}>0$ and $\lambda_{5}>0$ contradicts to (40).

2) $k_{d}^{*} \omega_{0}^{* 2}-k_{i}^{*} \geq 0$. If $\lambda_{0}=0$, then from (40) we have $\lambda_{1}+2 \lambda_{5} k_{p}^{*} \omega_{0}^{* 2}=0$, which in turn means that $\lambda_{1}>0$ and $\lambda_{5}<0$; otherwise, it is necessary that $\lambda=0$, which is a contradiction. Similarly, if $\lambda_{0}>0$, then from (38) we have $\lambda_{5}<0$. This again allows us to conclude from (40) that $\lambda_{1}>0$. Hence in either case, since $\lambda_{1}>0$, the constraint $g_{1}\left(k_{p}, k_{i}, k_{d}, \omega_{0}\right) \leq 0$ is active, i.e.,

$$
g_{1}\left(k_{p}^{*}, k_{i}^{*}, k_{d}^{*}, \omega_{0}^{*}\right)=p-k_{p}^{*}=0
$$

Likewise, that $\lambda_{5}<0$ implies that the equality constraint $h\left(k_{p}, k_{i}, k_{d}, \omega_{0}\right)=0$ is active. Solve these two equations yields

$$
\left(1-k_{d}^{*}\right) \omega_{0}^{* 2}=-k_{i}^{*}
$$

which clearly is a contradiction. In summary, we have shown that the solution $\lambda_{2}=\lambda_{3}=\lambda_{4}=0$ invariably leads to a contradiction and hence is not possible.
Consider then the case $\lambda_{3}=0, \lambda_{2}=\lambda_{4} \omega_{0}^{* 2}$. Clearly, neither $\lambda_{2}$ nor $\lambda_{4}$ can be equal to zero. In other words, $\lambda_{2}>0$, and $\lambda_{4}>0$. As a result, the constraints $g_{2}\left(k_{p}, k_{i}, k_{d}, \omega_{0}\right) \leq 0$ and $g_{4}\left(k_{p}, k_{i}, k_{d}, \omega_{0}\right) \leq 0$ are active and in turn $k_{d}^{*}=1, k_{i}^{*}=\sigma$. With $k_{d}^{*}=1$ and $k_{i}^{*}=\sigma$, we obtain from the constraint $h\left(k_{p}^{*}, k_{i}^{*}, k_{d}^{*}, \omega_{0}^{*}\right)=0$ the solution

$$
\omega_{0}^{* 2}=\frac{\sigma^{2}}{p^{2}-k_{p}^{* 2}+2 \sigma} .
$$

This establishes (36). Note that for $k_{p} \in\left(p, \sqrt{p^{2}+2 \sigma}\right)$, $\omega_{0} \in(\sqrt{\sigma / 2}, \infty)$.

ii) Proving the pseudo-concavity of $\hat{\tau}\left(k_{p}\right)$ Denote by $\hat{\tau}\left(k_{p}\right)=f\left(g\left(k_{p}\right)\right)$, where

$$
\omega_{0}=g\left(k_{p}\right)=\frac{\sigma}{\sqrt{p^{2}-k_{p}^{2}+2 \sigma}}
$$

and

$$
f\left(\omega_{0}\right)=\frac{\tan ^{-1} \frac{\omega_{0}}{p}}{\omega_{0}}+\frac{\tan ^{-1} \frac{\omega_{0}^{2}-\sigma}{\sqrt{\left(p^{2}+2 \sigma\right) \omega_{0}^{2}-\sigma^{2}}}}{\omega_{0}} .
$$

It follows that $\hat{\tau}^{\prime}\left(k_{p}\right)=f^{\prime}\left(\omega_{0}\right) g^{\prime}\left(k_{p}\right)$, with

$$
\begin{array}{r}
f^{\prime}\left(\omega_{0}\right)=\left(\frac{\frac{\omega_{0}}{p}}{1+\left(\frac{\omega_{0}}{p}\right)^{2}}-\tan ^{-1} \frac{\omega_{0}}{p}-\tan ^{-1} \phi\left(\omega_{0}\right)\right. \\
\left.+\frac{\phi^{\prime}\left(\omega_{0}\right) \omega_{0}}{1+\phi^{2}\left(\omega_{0}\right)}\right) \frac{1}{\omega_{0}^{2}}
\end{array}
$$

where

$$
\phi\left(\omega_{0}\right)=\frac{\omega_{0}^{2}-\sigma}{\sqrt{\left(p^{2}+2 \sigma\right) \omega_{0}^{2}-\sigma^{2}}}
$$

and

$$
\phi^{\prime}\left(\omega_{0}\right)=\frac{\left(p^{2}+2 \sigma\right) \omega_{0}^{3}+p^{2} \sigma \omega_{0}}{\left(\left(p^{2}+2 \sigma\right) \omega_{0}^{2}-\sigma^{2}\right)^{\frac{3}{2}}} .
$$

Clearly, $g^{\prime}\left(k_{p}\right)>0$. We prove below that a unique $\bar{k}_{p}$ exists such that $\hat{\tau}^{\prime}\left(k_{p}\right)>0$ for $p<k_{p}<\bar{k}_{p}$ and $\hat{\tau}^{\prime}\left(k_{p}\right)<0$ for $\bar{k}_{p}<k_{p}<\sqrt{p^{2}+2 \sigma}$, so as to prove the pseudo-concavity of $\hat{\tau}\left(k_{p}\right)$. Consider the function $\delta\left(\omega_{0}\right)=\omega_{0}^{2} f^{\prime}\left(\omega_{0}\right)$. We have $\delta\left(\omega_{0}\right) \rightarrow-\pi<0$ when $\omega_{0} \rightarrow \infty$. On the other hand, when $\omega_{0} \rightarrow \sqrt{\sigma / 2}$, we have

$$
\tan ^{-1} \frac{\omega_{0}}{p} \rightarrow-\tan ^{-1} \phi\left(\omega_{0}\right)
$$

which suggests that $\delta\left(\omega_{0}\right)>0$ as $\omega_{0} \rightarrow \sqrt{\sigma / 2}$. By the continuity of $\delta\left(\omega_{0}\right)$, there exists at least one $\bar{\omega}_{0}>\sqrt{\sigma / 2}$ such that $\delta\left(\bar{\omega}_{0}\right)=0$; correspondingly, there exists some $p<\bar{k}_{p}<\sqrt{p^{2}+2 \sigma}$ such that $\hat{\tau}^{\prime}\left(\bar{k}_{p}\right)=0$. Next, we prove that this $\bar{k}_{p}$ is unique, by showing that $\delta\left(\omega_{0}\right)$ is 
a monotonically decreasing function. Indeed, taking the derivative of $\delta\left(\omega_{0}\right)$ yields

$$
\delta^{\prime}\left(\omega_{0}\right)=-\frac{2}{p} \frac{\left(\frac{\omega_{0}}{p}\right)^{2}}{\left(1+\left(\frac{\omega_{0}}{p}\right)^{2}\right)^{2}}-\frac{\omega_{0} \theta\left(\omega_{0}\right)}{\left(1+\phi^{2}\left(\omega_{0}\right)\right)^{2}},
$$

where

$$
\theta\left(\omega_{0}\right)=2 \phi^{\prime 2}\left(\omega_{0}\right) \phi\left(\omega_{0}\right)-\left(1+\phi^{2}\left(\omega_{0}\right)\right) \phi^{\prime \prime}\left(\omega_{0}\right)
$$

and

$$
\phi^{\prime \prime}\left(\omega_{0}\right)=-\frac{\left(2 p^{2}+3 \sigma\right)\left(p^{2}+2 \sigma\right) \sigma \omega_{0}^{2}+p^{2} \sigma^{3}}{\left(\left(p^{2}+2 \sigma\right) \omega_{0}^{2}-\sigma^{2}\right)^{\frac{5}{2}}} .
$$

Rewrite

$$
\theta\left(\omega_{0}\right)=\frac{\omega_{0}^{4} \gamma\left(\omega_{0}\right)}{\left(\left(p^{2}+2 \sigma\right) \omega_{0}^{2}-\sigma^{2}\right)^{\frac{7}{2}}}
$$

where

$$
\begin{aligned}
\gamma\left(\omega_{0}\right)=2\left(p^{2}+2 \sigma\right)^{2} \omega_{0}^{4} & +\left(p^{2}+2 \sigma\right)\left(4 p^{2} \sigma+7 \sigma^{2}\right) \omega_{0}^{2} \\
& +\left(6 p^{4} \sigma^{2}+3 p^{2} \sigma^{3}+2 p^{6} \sigma\right) .
\end{aligned}
$$

Obviously, $\gamma\left(\omega_{0}\right)>0$, and so $\theta\left(\omega_{0}\right)>0$. As such, $\delta^{\prime}\left(\omega_{0}\right)<0$ and $\delta\left(\omega_{0}\right)$ is monotonically decreasing with $\omega_{0}$, and that the solution $\bar{\omega}_{0}$ to the equation $\delta\left(\omega_{0}\right)=0$ is unique. Since $g^{\prime}\left(k_{p}\right)>0$ and $\hat{\tau}^{\prime}\left(k_{p}\right)=\delta\left(\omega_{0}\right) g^{\prime}\left(k_{p}\right) / \omega_{0}^{2}$, the uniqueness of $\bar{k}_{p}$ is ascertained. We thus concludes that $\hat{\tau}\left(k_{p}\right)$ is pseudo-concave.

iii) Proving the monotonicity of $\bar{\tau}_{P I D}(\sigma)$ Let $\sigma_{1}<$ $\sigma_{2}$. From the above proof, $\bar{\tau}_{P I D}\left(\sigma_{1}\right)$ is uniquely achieved on the boundary $k_{i}^{*}=\sigma_{1}$ for $k_{i}$ in the interval $\left[\sigma_{1}, \infty\right)$. Since for any $\sigma_{2} \in\left(\sigma_{1}, \infty\right)$, i.e., $\sigma_{2}$ lies in the interior of $\left[\sigma_{1}, \infty\right)$,

$$
\begin{aligned}
\sup \left\{\tau\left(k_{p}, k_{i}, k_{d}\right):\left(k_{p}, k_{i}, k_{d}\right) \in \Xi\left(\sigma_{1}\right)\right\} \\
\quad>\sup \left\{\tau\left(k_{p}, k_{i}, k_{d}\right):\left(k_{p}, k_{i}, k_{d}\right) \in \Xi\left(\sigma_{2}\right)\right\} .
\end{aligned}
$$

Thus, $\bar{\tau}_{P I D}\left(\sigma_{1}\right)>\bar{\tau}_{P I D}\left(\sigma_{2}\right)$, that is, $\bar{\tau}_{P I D}(\sigma)$ is strictly decreasing with $\sigma>0$.

iv) The upper bound on $\bar{\tau}_{P I D}(\sigma)$ In view of (42), since for $\omega_{0}^{2} \geq \sigma$,

$$
\tan ^{-1} \frac{\omega_{0}-\frac{\sigma}{\omega_{0}}}{k_{p}} \leq \tan ^{-1} \frac{\omega_{0}}{k_{p}} \leq \tan ^{-1} \frac{\omega_{0}}{p},
$$

it follows that

$$
\hat{\tau}\left(k_{p}\right) \leq \frac{2 \tan ^{-1} \frac{\omega_{0}}{p}}{\omega_{0}}
$$

Furthermore, since $\frac{\tan ^{-1} \frac{\omega_{0}}{p}}{\omega_{0}}$ is monotonically decreasing with $\omega_{0}$, we have

$$
\sup _{\omega_{0}^{2} \geq \sigma} \hat{\tau}\left(k_{p}\right) \leq\left(\frac{\tan ^{-1} \frac{\sqrt{\sigma}}{p}}{\frac{\sqrt{\sigma}}{p}}\right) \frac{2}{p}
$$

For $\sigma / 2<\omega_{0}^{2}<\sigma$, since $\tan ^{-1}\left(\omega_{0}-\sigma / \omega_{0}\right) / k_{p}<0$, we have

$$
\hat{\tau}\left(k_{p}\right) \leq \frac{\tan ^{-1} \frac{\omega_{0}}{p}}{\omega_{0}} .
$$

The maximum of this upper bound is achieved at $\omega_{0}=$ $\sqrt{\sigma / 2}$, which leads to

$$
\sup _{\sigma / 2<\omega_{0}^{2}<\sigma} \hat{\tau}\left(k_{p}\right) \leq\left(\frac{\tan ^{-1} \frac{\sqrt{\frac{\sigma}{2}}}{p}}{\frac{\sqrt{\frac{\sigma}{2}}}{p}}\right) \frac{2}{p} .
$$

Taking the larger of the two upper bounds completes the proof.

\section{An illustrative example}

To illustrate the preceding results, we consider the delay plant (11) with $p=1$. For this plant, the maximal delay margin is $\bar{\tau}_{P I D}=2$. Select $\tau=1$. Tuning the PID controller gains as prescribed in (17) gives

$$
\begin{aligned}
& k_{d}=\left(-1+\frac{\sqrt{13}}{2}\right) e^{\frac{-5}{2}+\frac{\sqrt{13}}{2}}, \\
& k_{i}=\left(-\frac{263}{4}+\frac{73 \sqrt{13}}{4}\right) e^{\frac{-5}{2}+\frac{\sqrt{13}}{2}}, \\
& k_{p}=-\left(\frac{35}{2}-\frac{\sqrt{13}}{2}\right) e^{\frac{-5}{2}+\frac{\sqrt{13}}{2}},
\end{aligned}
$$

which results in the quadruple real closed-loop pole $s_{+}=$ $(-5+\sqrt{13}) / 2$. Obviously, $s_{+}$is a stable pole. Figure 4 exhibits the closed-loop spectrum distribution with the PID control gains given by (43). Figure 5 illustrates the relationship between $\tau$ selected in the PID design and the dominant pole $s_{+}$, which serves to show the tradeoff between the delay tolerable and the transient performance achieved, such as the decay rate of the closed-loop system. Note that different values of $p>0$ are examined. It is worth noting that the two quantities possess a monotone relationship. Figure 6 is helpful for assessing delay robustness, where different values of $p>0$ are also selected. Interestingly, in all the cases, and for any $\tau$ selected (equivalently, $s_{+}$is selected), the delay robustness range is greater than $\tau$, which means that with the MID design, one can guarantee at least the same amount of robustness range as $\tau$. Likewise, as $\tau$ increases, the dominant pole $s_{+}$becomes closer to the imaginary axis, but the delay robustness range $\bar{\tau}$ is increased. 


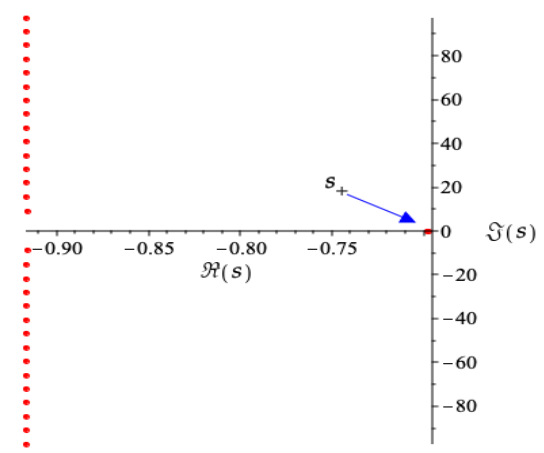

Figure 4. The distribution of the closed-loop spectrum with $\left(k_{p}, k_{i}, k_{d}\right)$ given by $(43)$, corresponding to $p=\tau=1$. The dominancy of $s_{+}=-\frac{5-\sqrt{13}}{2}$ is emphasized.

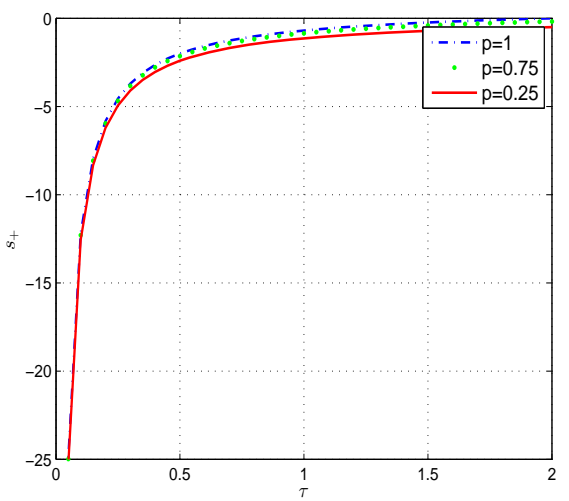

Figure 5. Variation of the dominant pole $s_{+}$by selection of $\tau<\bar{\tau}_{P I D}$.

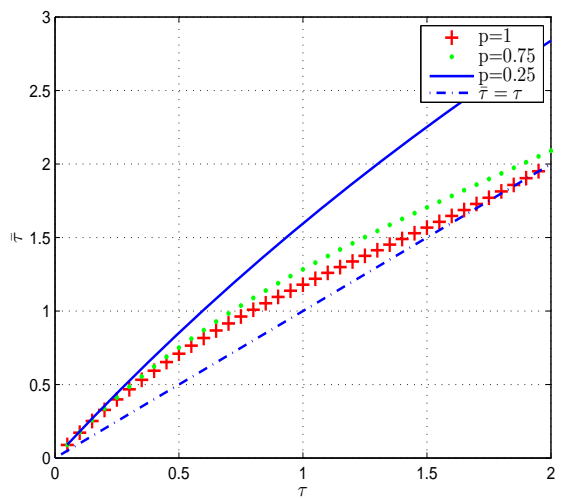

Figure 6. The delay margin achieved by the MID PID controller by selection of $\tau<\bar{\tau}_{P I D}$.

We next analyze the delay margin vs. steady-state tracking performance. Select $p=1.5$ and $\sigma=1$. Figure 7 demonstrates that $\hat{\tau}\left(k_{p}\right)$ is indeed a pseudo-concave function. Figure 8 plots the delay margin $\bar{\sigma}_{P I D}(\sigma)$ for $\sigma=0,0.2,0.5$ and 1 , with $p \in[0,3]$. It is clear that as $\sigma$ increases, the delay margin decreases. For different values of $\sigma$, the reduction of the delay margin, due to steady-state tracking specification, can be significant.

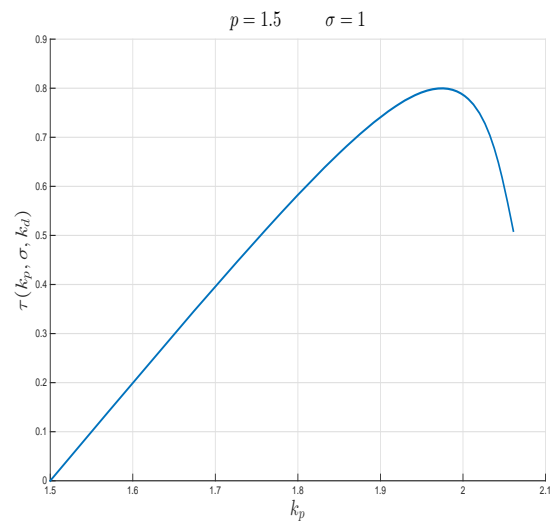

Figure 7. Pseudo-concavity of $\hat{\tau}\left(k_{p}\right)$.

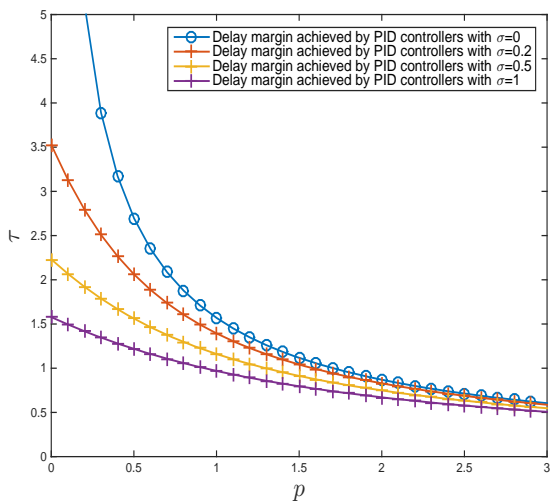

Figure 8. Delay margin vs. tracking error.

\section{Conclusion}

Pole placement of time-delay systems is a challenging problem which often defies accurate analysis and design. The difficulty becomes exacerbated when using PID control, due to its restricted degree of design freedom. Additionally, complexity may also arise for delay plants with relative degree less than two, since PID control will result in a neutral closed-loop delay system which may have infinitely many poles in the right half plane. This paper presents a case study where the difficulties are judiciously mitigated. We developed a MID design and tuning scheme for first-order unstable delay plants, which has been shown to possess desirable properties. Specifically, we show analytically that with this scheme, a real dominant closed-loop pole can be assigned precisely, thus guaranteeing the exponential decay rate of the system response. While achieving such transient performance, the MID design is also shown to be capable of maintaining a certain level of stability robustness against an uncertain delay. The tradeoff of the design, versus that of achieving delay margin and steady-state tracking performance, is analyzed. The latter is shown to be computable by solving a unimodal convex optimization problem. 
Extensions of this work may be pursued in several aspects. First, it is of immediate interest to contemplate on the problem of assigning dominant complex conjugate poles. This will further enrich the transient behavior of the closed-loop system, in gaining, e.g., a faster rise time and a shorter setting time. Likewise, it is of significant interest to seek extensions to second- and higherorder systems. Moreover, in practice, the implementation of the derivative control is often accompanied by a low-pass filter, resulting in the so-called filtered derivative control (Åström and Hägglund, 1995). It is of interest to develop a companion MID design and tuning rule for such practical implementation. For these purposes, it appears that the techniques employed herein, which draw heavily upon the theories of algebraic geometry and nonlinear programming, need to be generalized. Finally, it will be of interest to explore applications of the MID method to practical design problems. In this vein, we note that the MID method is in general applicable to linear time-invariant systems in which time delays may exist in the plant dynamics or control action is implemented via delayed feedback. Successful applications of this method have been reported in, e.g., the control of drilling systems (Auriol et al., 2021).

\section{Acknowledgments}

The authors are partially financially supported by NSFC under Grant 61973060, the Hong Kong RGC under Project CityU 11203120, a public grant overseen by the French National Research Agency (ANR) as part of the "Investissement d'Avenir" program, through the "iCODE Institute project" funded by the IDEX Paris-Saclay.

\section{References}

Åström, K.J. and Hägglund, T. (2004). Revisiting the Ziegler-Nichols step response method for PID control. Journal of Process Control, 14(6), 635-650.

Åström, K.J. and Hägglund, T. (1995). PID Controllers: Theory, Design, and Tuning, 2nd Ed. ISA.

Auriol, J., Boussaada, I., Mounier, H., and Niculescu, S.I. (2021). Torsional-vibrations Damping in Drilling Systems: Multiplicity-Induced-Dominancy-Based Design. In 24th International Symposium on Mathematical Theory of Networks and Systems. Cambridge, United Kingdom.

Bertsekas, D.P. (2016). Nonlinear Programming, 3rd Ed. Athena Scientific.

Boussaada, I. and Niculescu, S.I. (2016). Characterizing the codimension of zero singularities for time-delay systems. Acta Applicandae Mathematicae, 145(1), 4788.

Boussaada, I., Unal, H., and Niculescu, S.I. (2016). Multiplicity and stable varieties of time-delay systems: A missing link. In Proceeding of the 22nd International
Symposium on Mathematical Theory of Networks and Systems, 1-6.

Boussaada, I., Niculescu, S.I., El-Ati, A., Pérez-Ramos, R., and Trabelsi, K. (2020). Multiplicity-induceddominancy in parametric second-order delay differential equations: Analysis and application in control design. ESAIM: $C O C V, 26,57$.

Boussaada, I., Tliba, S., Niculescu, S.I., Ünal, H.U., and Vyhlídal, T. (2018). Further remarks on the effect of multiple spectral values on the dynamics of time-delay systems. application to the control of a mechanical system. Linear Algebra and its Applications, 542, 589604.

Boyd, S. and Vandenberghe, L. (2004). Convex Optimization. Cambridge University Press.

Bresch-Pietri, D., Chauvin, J., and Petit, N. (2012). Adaptive control scheme for uncertain time-delay systems. Automatica, 48(8), 1536-1552.

Chen, J., Fu, P., Méndez-Barrios, C.F., Niculescu, S.I., and Zhang, H. (2017). Stability analysis of polynomially dependent systems by eigenvalue perturbation. IEEE Transactions on Automatic Control, 62(11), 5915-5922.

Chen, J. and Chen, J. (2020). Tradeoff between delay margin and asymptotic tracking by PID control. In Proc. 2020 European Control Conference, 374-379. St. Petersburg, Russia.

Chen, J., Ma, D., Xu, Y., and Chen, J. (2019). Exact computation of delay margin by PID control: It suffices to solve a unimodal problem! In 2019 Chinese Control Conference, 242-249.

Das, S., Halder, K., and Gupta, A. (2020). Delay handling method in dominant pole placement based pid controller design. IEEE Transactions on Industrial Informatics, 16(2), 980-991.

Emre, D. and Mehmet, T.S. (2018). Digital PI-PD controller design for arbitrary order systems: Dominant pole placement approach. ISA Transactions, 79, 189201.

Firouzbahrami, M. and Nobakhti, A. (2017). Reliable computation of PID gain space for general secondorder time-delay systems. International Journal of Control, 90(10), 2124-2136.

Foias, C., Özbay, H., and Tannenbaum, A. (1996). Robust Control of Infinite Dimensional Systems: Frequency Domain Methods. Springer- Verlag.

Gaudette, D.L. and Miller, D.E. (2016). Adaptive stabilization of a class of time-varying systems with an uncertain delay. Mathematics of Control, Signals, and Systems, 28(3).

Hayes, N.D. (1950). Roots of the transcendental equation associated with a certain difference-differential equation. Journal of the London Mathematical Society, s1-25(3), 226-232.

Jaromír, F. and Pavel, Z. (2019). PID controller tuning via dominant pole placement in comparison with Ziegler-Nichols tuning. IFAC-PapersOnLine, 52(18), $43-48$. 
Ju, P. and Zhang, H. (2016). Further results on the achievable delay margin using LTI control. IEEE Transactions on Automatic Control, 61(10), 31343139.

Kress, R. (2014). Linear Integral Equations. SpringerVerlag, New York.

Luenberger, D.G. and Ye, Y. (1984). Linear and Nonlinear Programming, 2nd Ed. Springer.

Ma, D., Boussaada, I., Bonnet, C., Niculescu, S.I., and Chen, J. (2020a). Multiplicity-induced dominancy extended to neutral delay equations: Towards a systematic PID tuning based on rightmost root assignment. In Proc. 2020 American Control Conference, 16901695. Denver, CO.

Ma, D., Chen, J., Lu, R., Chen, J., and Chai, T. (2020b). Delay consensus margin of first-order multiagent systems with undirected graphs and PD protocols. IEEE Transactions on Automatic Control, 1-8. doi:10.1109/TAC.2020.3035556.

Ma, D. and Chen, J. (2019). Delay margin of low-order systems achievable by PID controllers. IEEE Transactions on Automatic Control, 64(5), 1958-1973.

Mangasarian, O.L. and Fromovitz, S. (1967). The Fritz John necessary optimality conditions in the presence of equality and inequality constraints. Journal of Mathematical Analysis and applications, 17(1), 37-47.

Michiels, W., Engelborghs, K., Vansevenant, P., and Roose, D. (2002). Continuous pole placement for delay equations. Automatica, 38(5), 747-761.

Middleton, R.H. and Miller, D.E. (2007). On the achievable delay margin using LTI control for unstable plants. IEEE Transactions on Automatic Control, 52(7), 1194-1207.

Miller, D.E. and Davison, D.E. (2005). Stabilization in the presence of an uncertain arbitrarily large delay. IEEE Transactions on Automatic Control, 50(8), 1074-1089.

O'Dwyer, A. (2013). Control of open-loop unstable processes with time delay using PI/PID controllers specified using tuning rules: An outline survey. In 24th IET Irish Signals and Systems Conference, 1-7.

Partington, J.R. and Bonnet, C. (2004). $H_{\infty}$ and BIBO stabilization of delay systems of neutral type. Systems and Control Letters, 52(3), 283-288.

Pavel, Z., Jaromir, F., and Tomas, V. (2013). Dimensional analysis approach to dominant three-pole placement in delayed PID control loops. Journal of Process Control, 23(8), 1063-1074.

Pólya, G. and Szegö, G. (1972). Problems and Theorems in Analysis, Vol. I: Series, Integral Calculus, Theory of Functions. Springer-Verlag, New York, Heidelberg, and Berlin.

Ramirez, A., Mondie, S., Garrido, R., and Sipahi, R. (2015). Design of proportional-integral-retarded (PIR) controllers for second-order LTI systems. IEEE Transactions on Automatic Control, (99), 1-6.

Rao, C.S., Santosh, S., and V, D.R. (2020). Tuning optimal PID controllers for open loop unstable first order plus time delay systems by minimizing ITAE cri- terion. IFAC-PapersOnLine, 53(1), 123-128.

Samad, T. (2017). A survey on industry impact and challenges thereof. IEEE Control Systems Magazine, 37(1), 17-18.

Silva, G.J., Datta, A., and Bhattacharyya, S.P. (2002). New results on the synthesis of PID controllers. IEEE Transactions on Automatic Control, 47(2), 241-252.

Srivastava, S. and Pandit, V.S. (2016). A PI/PID controller for time delay systems with desired closed loop time response and guaranteed gain and phase margins. Journal of Process Control, 37, 70-77.

Tavakoli, S. and Safaei, M. (2018). Analytical PID control design in time domain with performancerobustness trade-off. Electronics Letters, 54(13), 815817.

Thomas, G. and Ganesan, V. (2020). Optimal tuning of PID controller in time delay system: a review on various optimization techniques. Chemical Product and Process Modeling. doi:https://doi.org/10.1515/cppm2020-2001.

Vanbiervliet, J., Verheyden, K., Michiels, W., and Vandewalle, S. (2008). A nonsmooth optimisation approach for the stabilisation of time-delay systems. ESAIM: COCV, 14(3), 478-493.

Wang, H., Liu, J., Yu, X., Tan, S., and Zhang, Y. (2017). New results on PID controller design of discrete-time systems via pole placement. IFAC-PapersOnLine, 50(1), 6703-6708.

Xu, J., Zhang, H., and Xie, L. (2013). Input delay margin for consensusability of multi-agent systems. Automatica, 49(6), 1816-1820.

Zhang, W., Cui, Y., and Ding, X. (2020). An improved analytical tuning rule of a robust PID controller for integrating systems with time delay based on the multiple dominant pole-placement method. Symmetry, $12(9), 1449$.

Zhao, C. and Guo, L. (2017). PID controller design for second order nonlinear uncertain systems. Science China Information Sciences, 60(2), 022201.

Zhong, W.X. (2006). Duality System in Applied Mechanics and Optimal Control. Springer.

Zhou, B., Lin, Z., and Duan, G.R. (2009). Properties of the parametric lyapunov equation-based low-gain design with applications in stabilization of time-delay systems. IEEE Transactions on Automatic Control, 54(7), 1698-1704.

Zhou, B., Lin, Z., and Duan, G.R. (2012). Truncated predictor feedback for linear systems with long timevarying input delays. Automatica, 48(10), 2387-2399. 